\title{
The equation of state of fluid hydrogen at high density
}

G. CHABRIER

Laboratoire de Physique, Ecole Normale Supérieure de Lyon, 69964 Lyon Cedex07, France

\begin{abstract}
We present a free energy model for fluid hydrogen at high-density and hightemperature. This model aims at describing pressure dissociation and ionization, which occur in partially ionized plasmas encountered in the interiors of giant planets and low-mass stars. The model describes an interacting mixture of $\mathrm{H}_{2}, \mathrm{H}, \mathrm{H}^{+}$and $e^{-}$in chemical equilibrium. The concentrations of $\mathrm{H}^{2+}$ and $\mathrm{H}^{-}$ions are found to be negligible for equation of state purposes. Our model relies on the so-called chemical picture approach, based on the factorization of the partition function into translational, internal and configurational degrees of freedom. The present model is found to be unstable in the pressure-ionization regime and predicts the existence of a first-order plasma phase transition (PPT) which ends up at a critical point given by $T_{c}=15300 \mathrm{~K}, P_{c}=0.614 \mathrm{Mbar}$, and $\rho_{c}=0.35 \mathrm{gcm}^{-3}$. The transition occurs between a weakly ionized phase and a partially ionized $(\sim 50 \%)$ phase.
\end{abstract}

Nous présentons un modèle d'énergie libre pour l'hydrogène fluide à haute densité et haute température. Le but de ce modèle est de décrire la dissociation et l'ionisation en pression, telles qu'elles se produisent dans les plasmas partiellement ionisés rencontrés à l'intérieur des planètes géantes et des étoiles de faible masse. Le modèle décrit un fluide en interaction composé de $\mathrm{H}_{2}, \mathrm{H}, \mathrm{H}^{+}$et $e^{-}$en équilibre chimique. Les concentrations de $\mathrm{H}^{2+}$ et $\mathrm{H}^{-}$ sont négligeables pour les calculs d'équation d'état. Notre modèle repose sur 
l'approche chimique, basée sur la factorisation de la fonction de partition en degrés de liberté translationnel, interne et configurationnel. Le modèle présente une zone d'instabilité dans le domaine d'ionisation en pression et prédit l'existence d'une transition de phase plasma (PPT) du premier ordre, se terminant en un point critique donné par $T_{c}=15300 \mathrm{~K}, P_{c}=0.614$ Mbar, et $\rho_{c}=0.35 \mathrm{gcm}^{-3}$. La transition a lieu entre une phase faiblement ionisée et une phase partiellement ionisée $(\sim 50 \%)$.

\subsection{Introduction}

The similarities between hydrogen and alkali metals lead Wigner and Huntington (1935) to suggest that pressure-ionized hydrogen would behave like a monovalent 'metal' even at zero-temperature, and it has often been argued that first-order phase transition must occur between the two states, given the large difference between the molecular and the metallic states (Stevenson and Salpeter 1977; Ebeling and Richert 1985). Most of the recent investigations have focused on the zero-temperature or the room-temperature, where static compression experiments are now available above the megabar domain. These results indicate that a transition between a molecular and a semi-metal state occurs around 2 Mbar, but true metallization has not been observed unambiguously yet. This state is believed to occur around 2-3 Mbar.

Few models exist however at high-temperature, in the fluid range, where shock-wave experiments have clearly establish the stability of the fluid molecular phase up to $0.8 \mathrm{Mbar}$ (Nellis et al. 1984). Fluid hydrogen is the main component of stellar interiors and atmospheres. In most of astrophysical objects, hydrogen is ionized by temperature, but pressure ionization occurs in the interior of giant planets, brown dwarf stars and partially in the outermost layers of white dwarfs and low-masss stars. The recent discovery of global oscillations in Jupiter (see Mosser, these proceedings), as well as the recent achievements of helio- and astero-seismology (see Däppen and Fontaine, these proceedings) give us new information on the structure of these objects, and then on the properties of matter under extreme thermodynamic conditions. This stress the need for a correct calculation of fluid hydrogen at high-density, including a proper treatment of pressure dissociation and ionization. A simplified phase diagram of hydrogen at high temperature is shown on Figure 1, with the internal temperature profiles of a few dense astrophysical objects.

In section II, we give a short presentation of our free energy model, which has been presented extensively elsewhere (Saumon and Chabrier 1991, 


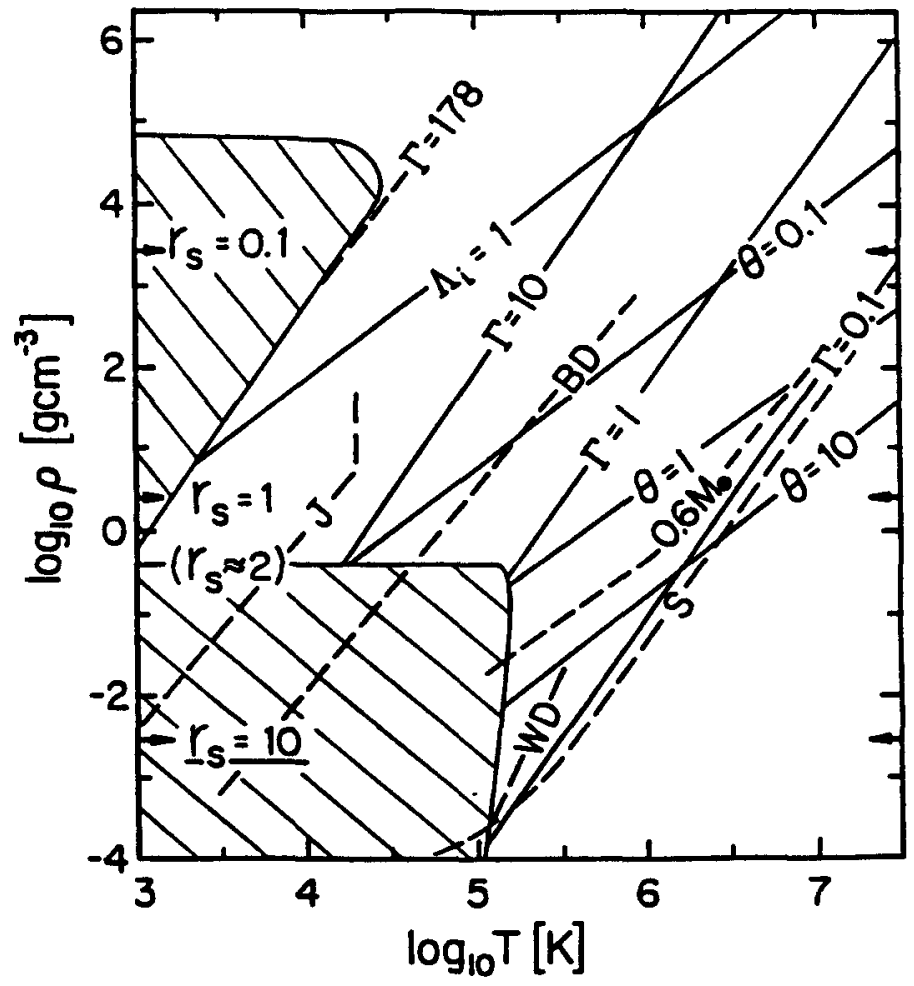

Fig. 13.1 Simplified $(\rho, T)$ phase diagram for hydrogen. A few physical regimes are identified : above the line $\Gamma=1$, where $\Gamma$ is the usual coupling parameter, the classical ionic plasma is strongly coupled whereas correlations are dominant in the quantum electron plasma below the line $r_{8}=1$, where $r$, is the mean inter-electronic distance in unit of Bohr radius. Electrons are degenerate above the line $\theta=k T / \epsilon_{j}=1$, where $\epsilon_{F}$ is the electron Fermi energy. Protons are classical below the line $\Lambda_{i}=1$, where $\Lambda_{i}$ is the De Broglie wavelength in unit of the mean inter-ionic distance $a$. The curve $\Gamma=178$ denotes the crystallization line of the $\mathrm{H}^{+}$plasma. The various temperature profiles are characteritic of the interior of Jupiter (J), a brown dwarf (BD), a ZZ-Ceti white dwarf (WD) and the Sun (S).

1992). In section III, we discuss in detail the PPT predicted by our model whereas a comparison with other models is discussed in Section IV. Section $\mathrm{V}$ is devoted to the astrophysical applications and Section VI to the conclusion.

\subsection{Description of the free energy model}

The model relies on the so-called chemical picture, in the sense that we assume the existence of independent, bound configurations such as $\mathrm{H}$ atoms, $\mathrm{H}_{2}$ molecules, interacting with pair potentials. At densities corresponding to pressure ionization, such a scheme is erroneous and the concept 
of individual pair potential fails, requiring the use of quantum-statistical many-body theory, i.e. a physical picture where only fundamental particles (electrons and nuclei) exist (see the reviews by Rogers, Alastuey and Perrot and Dharma-wardana). In particular, our approach does not take into account the possibility of excitonic states, i.e. clusters or pseudo-atoms and pseudo-molecules, as described in Perrot and Dharma-wardana (these proceedings). Although formally exact, the physical picture, however, involves either diagramatic expansion which converge only at low-density or hightemperature (see the reviews by Rogers and Alastuey), or, when extended to higher-density, involve a coupled treatment of classical and quantummechanical N-body theories, which renders practical applications for the calculation of astrophysical EOS nearly impossible. In view of these difficulties, the chemical picture, and its inherent factorization of the partiction function in terms of different particle interactions, remains a very powerful method. It can be view as the best compromise between the rigorous treatment and the practical application. It is why it is important to compare the results obtained with "chemical" models with the ones derived from "physical" ones.

Our EOS consists of a general free energy model which applies in the regime of partial temperature- or pressure-ionization, and which reduces to a so-called "neutral" model and "plasma" model respectively at lowdensity/low-temperature and at high-density and/or high-temperature.

\subsubsection{Model for neutral hydrogen}

At low-density $\left(\rho \lesssim 1 \mathrm{gcm}^{-3}\right.$ ), low temperature $\left(\mathrm{T} \lesssim 10^{4} \mathrm{~K}\right.$ ), hydrogen is adequately described as a mixture of $\mathrm{H}$ atoms and $\mathrm{H}_{2}$ molecules. The concentrations of $\mathrm{H}^{-}$and $\mathrm{H}^{2+}$ ions are found to be negligible $\left(<10^{-3}\right)$ for EOS purposes. Because all particles are very nearly classical in this regime, we can factorize the partition function and treat the small quantum effects with a semi-classical approximation. If we make the additional assumption that the internal levels of atoms and molecules are only weakly affected by the presence of nearby particles, as suggested by available experimental results up to electronic densities as large as $N_{e} / V \sim 10^{21} \mathrm{~cm}^{-3}$ (Weise et al. 1972, Grabowski et al. 1987, Hashimoto and Yamaguchi 1983), the Helmoltz free energy separates into ideal, configurational, internal, and quantum contributions :

$$
F\left(N_{H}, N_{H_{2}}, V, T\right)=F_{i d}+F_{c o n f}+F_{\text {int }}+F_{q m}
$$




\subsubsection{1 the configuration term}

This term arises from the interaction between the different particles in their ground state. Computation of these interactions requires the knowledge of three interaction potentials $\phi_{\mathrm{H}_{2}-\mathrm{H}_{2}}, \phi_{\mathrm{H}-\mathrm{H}}$ and $\phi_{\mathrm{H}_{2}-\mathrm{H}}$. For $\phi_{\mathrm{H}_{2}-\mathrm{H}_{2}}$ we use an effective potential derived from shock tube experiments (Nellis et al. 1984; Ross et al. 1983) which implicitly includes many-body effects. Since no similar experimental data exist for $\phi_{H-H}$ and $\phi_{H_{2}-H}$, we have used ab-initio potentials (Kolos and Wolniewicz 1965; Porter and Karplus 1964). We treat the spin dependence of the $\mathrm{H}-\mathrm{H}$ interaction by averaging the interaction potentials of the singlet and triplet states; the resulting $\mathrm{H}-\mathrm{H}$ potential has no bound states. The three potentials have been fitted by generalized Morse potentials.

The configuration free energy $F_{\text {conf }}$ is derived from thess interaction potentials in the framework of the WCA fluid perturbation expansion (Weeks, Chandler and Andersen 1971). In this theory, the interaction potential is split into a repulsive reference potential $\phi^{r e f}(r)$ and a weak, attractive perturbation potential $\phi^{\text {pert }}(r)$. We approximate the free energy of the reference system by that of a hard sphere fluid, which is known analytically (Mansoori et al. 1975), whereas the contribution of the perturbation potential is given by the first term of the free energy expansion (High Temperature Approximation) :

$$
\begin{aligned}
& F_{\text {conf }}(N, V, T)=F_{H S}\left(N_{1}, N_{2}, \sigma_{1}, \sigma_{2}, V, T\right) \\
& +\frac{1}{2 V} \sum_{\alpha, \beta=1}^{2} N_{\alpha} N_{\beta} \int \phi_{\alpha, \beta}^{\text {pert }}(r) g_{\alpha, \beta}^{H S}(r) d \vec{r}
\end{aligned}
$$

Here the $g_{\alpha, \beta}(r)$ are the hard sphere pair correlation functions (Gründke and Henderson 1972) and $\sigma_{1}$ and $\sigma_{2}$ are the density and temperature-dependent hard sphere diameters determined thermodynamically by the WCA criterion (Weeks, Chandler and Andersen 1971). The standard WCA scheme, derived originally for liquid state theory, has been extended for this particular approach to high-density and high-temperature (Saumon, Chabrier and Weis 1989). The excess internal energy and pressure derived from this expansion scheme agree within less than $3 \%$ with MC simulations for the density and temperature range of interest (Saumon, Chabrier and Weis 1989). This assesses the validity of the configuration energy (2) for the $\mathrm{H}-\mathrm{H}_{2}$ mixture. 


\subsubsection{The Internal Free Energy}

The effect of near-neighbor interactions on the internal structure of bound species is essential to a correct description of pressure dissociation and ionization. We have used in our model the so-called occupation probability formalism (OPF) derived by Hümmer and Mihalas (1988). In this formalism, the internal free energy reads :

$$
F_{i n t}=-k T L n \sum_{\alpha=1}^{2} N_{\alpha} \sum_{i} \omega_{\alpha i} g_{\alpha i} e^{-\epsilon_{\alpha i} / k T}
$$

where $i$ runs over all internal states of species $\alpha$ and $\omega_{\alpha i}, g_{\alpha i}$ and $\epsilon_{\alpha i}$ are respectively the occupation probability, the multiplicity and the unperturbed energy of state $i$. The density dependent $\omega_{\alpha i}$ are computed from the configuration term $F_{\text {conf }}$ in the free energy. This ensures consistency of both the interactions and their effects on the IPF. It also provides a smooth cut-off of the IPF, and therefore a plausible pressure dissociation/ionization effect. Moreover the present method uses unperturbed energy eigenvalues and does not invoke hypothetical energy level shifts of doubtful validity. As a matter of fact, such shifts have been shown to be too small to be significant, as mentioned above (Wiese et al. 1972, Grabowski et al. 1987, Hashimoto and Yamaguchi 1983).

In practice, however, one must resort to a linearization of $F_{\text {conf }}$ to compute the occupation probability (see Hümmer and Mihalas (1988) for details). Calculations including terms beyond the density-linear term have been computed recently for helium at high-density (Aparicio and Chabrier 1994). In addition our occupation probabilities include neutral particle interaction only. The effect of charged particles, i.e. Stark ionization, requires a knowledge of the plasma microfield distribution at high-density, which complicates tremendously the calculations. The effect of the microfield will be discussed in detail later in the paper.

In our treatment of the IPF of $\mathrm{H}_{2}$, we have included all vibrational and rotational levels of each bound state of the molecule (Hüber and Herzberg 1979).

The term $F_{q m}$ in Eq.(1) is the quantum contribution to the free energy, which is always a weak perturbation of the classical free energy in the domain of interest for the hydrogen EOS, and then has been calculated to the first non-vanishing order in the Wigner-Kirkwood $\hbar^{2}$ expansion. 


\subsubsection{Model for fully ionized hydrogen}

For $k T \gtrsim 1$ Ryd or $\rho \gtrsim 2 \mathrm{gcm}^{-3}$ (corresponding to $r_{s} \sim 1$, where $r_{s}$ is the mean inter-electronic spacing in units of Bohr radius $a_{o}$ ), the fluid is a fully ionized electron-proton plasma. At these densities, the mean electron-ion potential energy $E_{i e}=e^{2} / r_{s} a_{o}$ is smaller than the electron Fermi energy $E_{F}$ so that the plasma can be described as a superposition of an electronscreened ionic fluid and a rigid electron background (Ashcroft and Stroud 1978). Under these conditions, the free energy of the plasma reads :

$$
F=F_{i d}-N k T \ln \int e^{\beta U_{e f f}(r)} d \vec{r}+F_{x c}+F_{q m}
$$

where $F_{i d}$ denotes the ionic and electronic perfect gas contributions, $F_{x c}$ is the exchange and correlation free energy of the electron gas at finite temperature (Ichimaru et al. 1987). The second term on the r.h.s of equation (4) is the free energy of the screened ionic fluid, calculated within the framework of the hyper-netted chain (H.N.C) theory for the temperature and density dependent screened Coulomb potential $U_{\text {eff }}(k, V, T)=4 \pi(Z e)^{2} / k^{2} \epsilon(k, V, T)$ (Chabrier 1990). The dielectric function $\epsilon$ is the finite temperature Lindhard function corrected with a local field correction for the short-range interaction between electrons (Utsumi and Ichimaru 1982). The model free energy (4) shows excellent agreement with existing Monte-Carlo calculations at finite and zero-temperature and with non-adiabatic calculations (see Chabrier 1990 for details).

At very low-density and high-temperature, the electrons behave almost classically and the thermodynamics of the electron-proton plasma can be evaluated by the Debye-Huckel two-component limit, corrected for small degeneracy effects (DeWitt 1962) :

$$
\left(\frac{F^{e x}}{N k T}\right)_{\rho \rightarrow 0} \approx \frac{1}{\sqrt{3}} \Gamma^{3 / 2}\left(Z_{i}+1\right)^{1 / 2}<Z^{2}>^{2 / 3}\left(1-\frac{3 \pi^{1 / 2}}{2^{7 / 2}} \gamma_{e}+\ldots\right)
$$

where $\left\langle Z^{2}\right\rangle=\sum_{\alpha} x_{\alpha} Z_{\alpha}^{2}, \alpha$ denoting the ionic and electronic species. The first term in Eq.(5) is the leading term of the classical cluster expansion, i.e. the Debye term, whereas the second term in the bracket represents the correction for electron quantum effects, given by the electron quantum diffraction parameter $\gamma_{e}=\frac{1}{k T}\left(2 \pi \hbar^{2} n_{e} e^{2} / m_{e}\right)$.

At intermediate densities, the free energy is interpolated smoothly between the high-density model (4) and the low-density model (5). The quantum correction $F_{q m}$ for the ions is also calculated to leading order in $\hbar^{2}$, using a Wigner-Kirkwood expansion for the screened potential $U_{\text {eff }}$. 


\subsubsection{Model for partial ionization}

The two models described above are combined to give a description of the thermodynamics of the plasma in the partial ionization zone. The interaction between charged and neutral particles in their ground state is treated through a polarization potential approach (Kraeft et al. 1986). The twobody potential is approximated by an interpolation between a hard-core repulsion at short distance and a screened dipolar potential outside the core :

$$
V_{i}^{p o l}(r)=-\frac{e^{2} \alpha_{i}}{2}\left[\frac{1+r \kappa}{r^{2}+R_{i}^{2}}\right]^{2} e^{-2 \kappa r}
$$

The hard core radii $R_{i}$ are chosen to be the radii for $\mathrm{H}$ and $\mathrm{H}_{2}$ obtained for the configuration energy from the WCA criterion. $\alpha_{i}$ denotes the polarizability of species $i$, and $\kappa$ is the inverse screening length of the electron-ion plasma, which enters the screened potential $U_{\text {eff }}$ in Eq.(4) (see Saumon and Chabrier 1992 for details). The hard core contribution effectively reduces the volume available for the ionic and electronic ideal terms by a factor $(1-\eta)$ where $\eta$ is the hard core packing fraction for $\mathrm{H}$ and $\mathrm{H}_{2}$. The second contribution introduces an additional polarization term $F_{\text {pol }}$ to the free energy given by :

$$
F_{p o l}=4 k T \frac{N_{e^{-}}}{V} \sum_{\alpha=1}^{2} N_{\alpha} B_{\alpha i}
$$

The $B_{\alpha i}$ denote the virial coefficients of particle of species $\alpha$, i.e. :

$$
B_{i, e^{-}}=B_{i, H^{+}} \equiv B_{i}=2 \pi \int_{R_{i}}^{\infty}\left(1-e^{-\beta V_{i}^{p o l}(r)}\right) r^{2} d r
$$

The general model free energy for the hydrogen fluid finally reads:

$$
\begin{aligned}
F\left(V, T, N_{H_{2}}, N_{H}, N_{H^{+}}, N_{e^{-}}\right) & =F_{i d}\left(V, T, N_{H_{2}}, N_{H}\right) \\
& +F_{i d}\left((1-\eta) V, T, N_{H^{+}}, N_{e^{-}}\right) \\
& +F_{N}^{e x}\left(V, T, N_{H_{2}}, N_{H}\right) \\
& +F_{I}^{e x}\left(V, T, N_{H^{+}}, N_{e^{-}}\right) \\
& +F_{p o l}\left(V, T, N_{H_{2}}, N_{H}, N_{H^{+}}, N_{e^{-}}\right)
\end{aligned}
$$

where the subscript " $i d$ " denotes the ideal contribution whereas $F_{N}^{e x}$ and $F_{I}^{e x}$ stand for the non-ideal contributions of the neutral model and the 


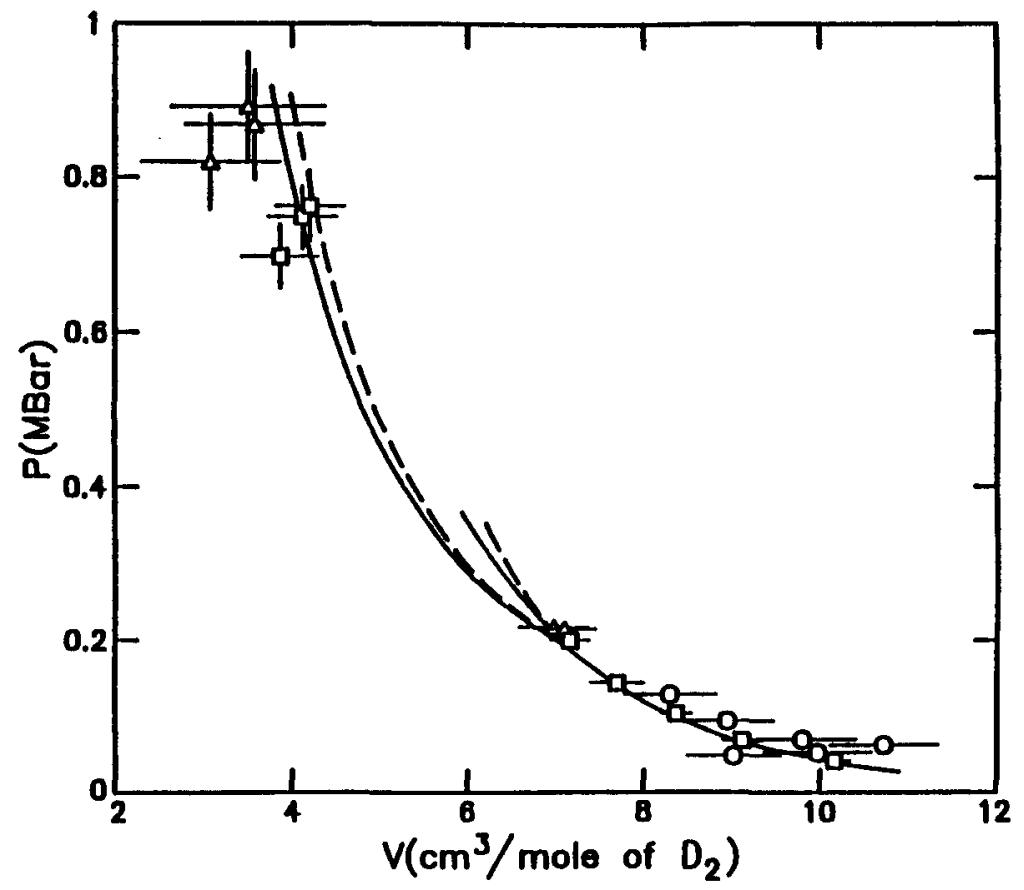

Fig. 13.2 Single and double-shock Hugoniot curves of $\mathrm{D}_{2}$. The solid curves shows the theoretical Hugoniot curves derived from the model free energy (9) after suitable modification for deuterium. The squares, circles and triangles denot the experimental data.

fully ionized model developed in the previous sections, respectively. Having imposed the electroneutrality condition, we minimize the free energy (9) at fixed total density and temperature to obtain the chemical equilibrium of the four component mixture $\left(\mathrm{H}_{2}, \mathrm{H}, \mathrm{H}^{+}, \mathrm{e}^{-}\right)$:

$$
\left.\frac{\partial \tilde{F}}{\partial x_{1}}\right|_{x_{2}, \rho, T}=\left.\frac{\partial \tilde{F}}{\partial x_{2}}\right|_{x_{1}, \rho, T}=0
$$

where $\tilde{F} \equiv \tilde{F}\left(x_{1}, x_{2}, \rho, T\right)$ is the specific free energy per proton, $x_{1}=x_{H}$, $x_{2}=x_{H_{2}}$ and $\rho$ is the mass density.

\subsection{Results and discussion}

Figure 2 shows the theoretical Hugoniot curves derived from our model for the single and double-shock experiments on $H$ and $D_{2}$ respectively along with the experimental data. In view of the experimental uncertainty, the agreement is excellent.

We calculated the limit of stability of the model free energy (9) as a function of the density along several isotherms and found a first order phase 
transition $(\partial P / \partial \rho<0)$ in the regime of pressure ionization. The transition is associated with a sudden, discontinuous shift of the chemical equilibrium toward a high degree of ionization. We emphasize that this phase change occurs strickly as a result of the minimization of our free energy model. It does not arise from any additional assumption. We have accordingly studied the phase equilibrium in the regime of pressure ionization systematically (Saumon and Chabrier 1992) :

$$
T^{I}=T^{I I} ; P^{I}=P^{I I} ; \mu_{H}^{I}(P, T)=\mu_{H}^{I I}(P, T)
$$

The characteristic of the coexistence curve are given in Table I. The phase transition ends in a critical point whose coordonates are : $P_{c}=0.614 \mathrm{Mbar}$, $\rho_{c}=0.347 \mathrm{gcm}^{-3}$ and $T_{c}=15300 \mathrm{~K}$. The slope of the coexistence curve $d P / d T$ is negative, which is consistent with the positive entropy discontinuity $\delta S=S^{I I}-S^{I}$, a likely consequence of the increasing contribution of thermal effects at higher temperature, and the larger entropy in the plasma phase than in the molecular phase.

Figure 3 shows the concentration of molecules and charged particles as a function of density for a few isotherms. We draw the following conclusions:

i) The system undergoes a first-order phase transition from a neutral phase $\left(x_{e^{-}} \leq 10^{-2}\right.$ for $\left.T<T_{c}\right)$ to a partially ionized phase $\left(x_{e^{-}} \approx 0.5\right)$ as density increases.

ii) At the transition pressure, the degree of ionization increases discontinuously whereas the concentration of molecules drops drastically, indicating that molecular dissociation and pressure ionization occur at almost the same density. Pressure ionization does not occur by first dissociating the molecules into atomic hydrogen as believed usually.

iii) Above the critical density, the system reaches complete ionization very gradually. This points out the qualitative difference found when treating pressure ionization with realistic potentials and with pure hard sphere interactions (Ebeling and Richert 1985). Even though the model for the neutral species is highly questionable above the transition density, we believe these qualitative features to be physicaly realistic.

iv) The first-order phase transition persists even when no coupling exists between the neutral and the fully ionized models. This indicates that the source of the PPT does not lie in the interaction between neutral and charged particles but rather in the very nature of the difference of interactions in the respective neutral and plasma phase. We actually believe the PPT to be due to the large difference between the strongly repulsive, hardsphere type potential in the insulating, molecular phase and the smoother 
Table 13.1. Characteristic of the plasma phase transition. For each temperature, we give the transition pressure, the density and the ionization fraction for each phase. The change in entropy is $\Delta S=S^{I I}-S^{I}$.

\begin{tabular}{lllllll}
\hline \hline $\begin{array}{l}\log T \\
(\mathrm{~K})\end{array}$ & $\begin{array}{l}\mathrm{P} \\
\text { (Mbar) }\end{array}$ & $\begin{array}{l}\rho^{I} \\
\mathrm{gcm}^{-3}\end{array}$ & $\begin{array}{l}\rho^{I I} \\
\mathrm{gcm}^{-3}\end{array}$ & $\begin{array}{l}2 x_{H^{+}}^{I} \\
\times 10^{-3}\end{array}$ & $2 x_{H^{+}}^{I I}$ & $\begin{array}{l}\Delta S \\
\mathrm{k}_{B} / \text { proton }\end{array}$ \\
\hline 3.70 & 2.14 & 0.75 & 0.92 & 1.4 & 0.48 & 0.615 \\
3.78 & 1.95 & 0.70 & 0.88 & 2.1 & 0.50 & 0.590 \\
3.86 & 1.62 & 0.64 & 0.80 & 3.0 & 0.50 & 0.544 \\
3.94 & 1.39 & 0.58 & 0.74 & 5.1 & 0.51 & 0.508 \\
4.02 & 1.13 & 0.51 & 0.65 & 8.8 & 0.52 & 0.464 \\
4.10 & 0.895 & 0.43 & 0.55 & 20. & 0.50 & 0.421 \\
4.18 & 0.631 & 0.35 & 0.38 & 170. & 0.33 & 0.142 \\
4.185 & 0.614 & 0.35 & 0.35 & 180. & 0.18 & 0 \\
\hline \hline
\end{tabular}

Yukawa-type potential in the conducting, plasma phase. This is supported by the fact that the PPT disappears above a temperature for which the dominant species in the neutral phase is no longer molecular hydrogen but atomic hydrogen, since the repusive part of the $\mathrm{H}-\mathrm{H}$ potential is very similar to the screened inter-ionic potential (see Saumon and Chabrier 1992). In this sense, the PPT can be related to the metal-insulator transition in metals associated with the liquid-vapor transition. The crucial difference between the two effective interactions in the metallic and insulating phases, which reflects the change of the nature of the electronic states, leads eventually to a polarization catastrophe and the impending insulator-metal transition (Goldstein and Ashcroft 1985).

\subsubsection{Uncertainties in the plasma phase transition}

In order to examine importance of some physical inputs of our model on the final results, we have carried out a limited set of calculations of the PPT using variations on our free energy model. These various PPT results are summarized on Figure 4, where the solid curve is the PPT derived from the basic model (9).

\subsubsection{Influence of coupling}

The most basic approximation in our model is to forbid the neutral and the charged particles to mix. This corresponds to a simple comparison of the two free energies (1) and (4). The resulting phase transition is what 


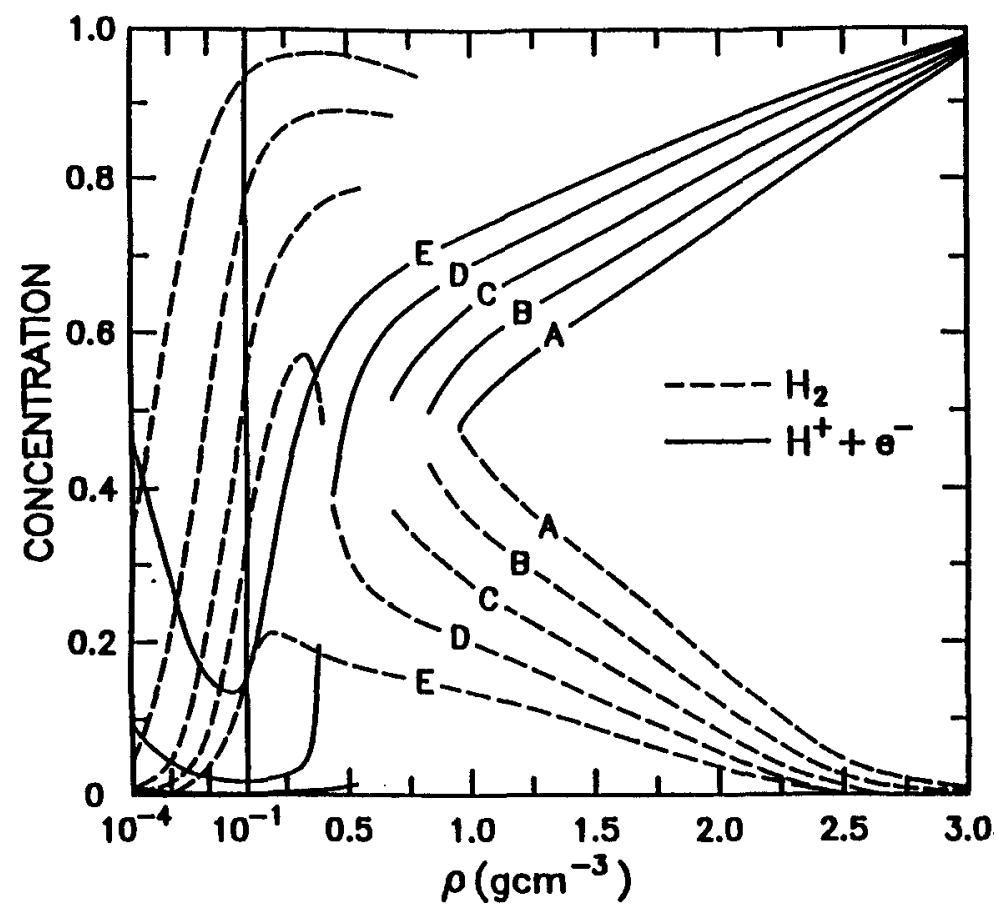

Fig. 13.3 Concentration of $\mathrm{H}_{2}$ and of charged particles $\left(\mathrm{H}^{+}+e^{-}\right)$near the PPT. Isotherms are labeled according to $\log T=A, 3.70 ; B, 3.86 ; C, 4.02$; $D, 4.18 ; E, 4.34$. The left panel shows the low-density behavior on a logdensity scale.

we call the "forced" transition. It is shown by the dashed line in Fig. 4. The transition line lies at $P \approx 3.2 \mathrm{Mbar}$ and is nearly independent of the temperature.

A more realistic variation on our final model is indicated by the triangles in Fig. 4. For these calculations, the PPT is computed without any coupling between the two models (1) and (4). In this approximation, plasma and neutral particles are allowed to mix but the two fluids do not interact. This corresponds to the free energy model (9) with $\eta=0$ and $F_{p o l}=0$. The highest temperature point shown for this calculation $\left(\log T_{c}=4.215\right)$ indicates the corresponding critical point. As already mentioned, it is important to stress that the first-order phase transition persists in this approximation, when no coupling exists between the neutral and the fully ionized models, indicating that the source of the PPT does not lie in the interaction between neutral and charged particles. The next variation is provided using a different expression for the screening length of the plasma so that the polarization free energy $F_{\text {pol }}$ is increased by about a factor of 5 . This results in the coex- 


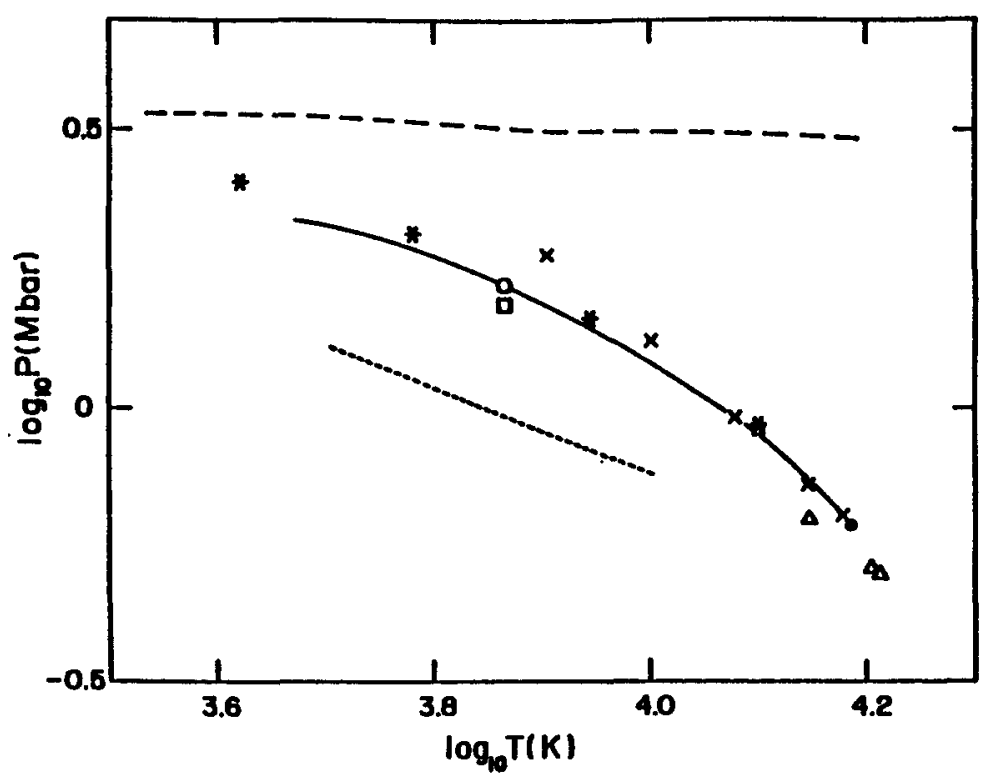

Fig. 13.4 The effects of various assumptions in the free energy model upon the plasma phase transition. The solid and dashed curves are coexistence curves for the PPT and for the forced phase transition, respectively. The crosses $(x)$ indicate the PPT obtained in a calculation where $F_{\text {pol }}$ (Eq. (7)) was overestimated by a factor of 5 . The dotted line shows the coexistence curve obtained when using the polarization radius of Redmer et al. (1987) in $F_{\text {pol }}$. When softened interaction potentials are used, the PPT occurs at higher pressures, as shown by the asterisks (*). When no coupling is allowed, the transition is shown by triangles $(\Delta)$. The open circle (o) indicates the effect of softening the lowest vibrational frequency of the $\mathrm{H}_{2}$ molecule by $4 \%$. See text for details.

istence curve indicated by crosses, with the critical point at $\log T_{c}=4.175$. The fourth variation is to use the polarisation radius for atomic $\mathrm{H}$ determined by Redmer, Röpke and Zimmermann (1987), $R_{H}=1.4565$ a.u. The $\mathrm{H}_{2}$ polarization radius was estimated by scaling the $\mathrm{H}$ value with polarizability : $R_{H_{2}} \approx R_{H}\left(\alpha_{H_{2}} / \alpha_{H}\right)^{1 / 3} \approx 1.55$ a.u. These radii are about $50 \%$ smaller than the ones used in the original calculations, increasing $F_{\text {pol }}$ by about one order of magnitude. The free energy model still undergoes unstabilities and the resulting phase line is shown on Fig. 4 by the dotted line. The transition pressure is lowered by about $40 \%$. The new critical point lies near $\log T_{c}=4.0$ and $P_{c}=0.76 \mathrm{Mbar}$. This is from far the most important variation in our results.

We have examined ex post facto the effect of Stark ionization as a form of coupling between the neutral and the ionized free energy models on our 
results. The effect of the plasma microfield is estimated by modifying the occupation probability in introducing a Holtzmark distribution. This is reasonnable in the low-density phase where the concentration of charged particles remains small, as discussed above. We find the effect to be negligible for $\log T \leq 4.1$. The importance rises rapidly near the critical point, however, following the increase in degree of ionization.

\subsubsection{2 $\mathrm{H}_{2}$ internal partition function}

In this section we examined the effect of the recently observed softening of the vibron frequency in the IPF of the $\mathrm{H}_{2}$ molecule (Hemley and Mao 1988). We have recomputed the transition pressure with the model given by Eq.(9) after reducing the vibration constant $W_{e}$ in the IPF of $\mathrm{H}_{2}$ by $4 \%$, as suggested by the experiment. This raises the transition pressure by $1 \%$, as shown by the open circle in Fig.4.

An other important issue is the rotational partition function of $\mathrm{H}_{2}$. The asymetry of the $\mathrm{H}_{2}-\mathrm{H}_{2}$ potential is expected to hinder rotation at highpressure, which can be interpreted as an increase of the rotational temperature $\theta_{\text {rot }}$. That could decrease substantially the entropy of the molecular phase and affect the location of the PPT. Our treatment uses the rotation temperature of the free molecule $(\approx 85 \mathrm{~K})$ at all densities. Recent experiments (Hemley, Mao and Shu 1990) show that the $\mathrm{H}_{2}$ molecules undergo significant rotational motion up to $1.6 \mathrm{Mbar}$ at $77 \mathrm{~K}$, a pressure typical of the PPT coexistence curve. Interestingly, the Raman spectrum of the roton mode does not show any line shifts of consequence for the EOS : $\theta_{\text {rot }}$ appears to be nearly independent of pressure, well above the PPT. Moreover, thermal effects are more important in our calculations so that we can expect rotational modes to freeze at much higher temperatures than is observed experimentally below room temperature.

\subsubsection{Softening of the potential}

Recently, Hemley et al. (1990) derived a new experimentally determined $\mathrm{H}_{2}-\mathrm{H}_{2}$ potential based on X-ray diffraction measurements of solid $\mathrm{H}_{2}$ at $\mathrm{T}=300 \mathrm{~K}$ and $P \lessgtr 0.26 \mathrm{Mbar}$. This potential is slighly softer than the potential used in our calculations (Ross et al. 1983; Saumon and Chabrier 1991). Given the lack of information for the $\mathrm{H}-\mathrm{H}$ and $\mathrm{H}_{2}-\mathrm{H}$ potentials at high density, we mimicked many-body effects by softening arbitrarily the repulsive part of the potentials respectively by $20 \%$ and $35 \%$. Results are shown by the asterisks in Fig.4. In all cases, the pressure and the density of transition increase slightly, as expected for a softened EOS in phase I. The 
qualitative features of he PPT are not affected, and the magnitude of the effect is less than $5 \%$.

\subsubsection{Strong ion-electron coupling}

Our free energy model in the fully ionized phase is based on the so-called linear response theory, which is valid as long as the ion-electron interaction is negligible compared with the ion-ion interaction, or the electron-ion potential energy is negligible compared with the electron kinetic energy. This fails at low density, where the electrons become strongly correlated, and where electron localization begins. We expect the ion-electron non-linear effects in the plasma phase to lower slightly the free energy of the plasma (by a few percents around the critical point), favoring ionization. The same effect is expected from a proper inclusion of micro-field effects on the excited states. Table II shows preliminary comparison between the present calculations and calculations by Perrot and Dharma-wardana (these proceedings) based on the so-called density-functional theory, where ion-electron interactions in the plasma are treated beyond the linear response. As expected the degree of ionization is underestimated in our calculations. However the pressure and the entropy obtained within the two formalisms are in very good agreement ( $\$ 3 \%$ ). This adds credibility to the present EOS for dense hydrogen since the results are based on two completely different type of calculations.

\subsubsection{Band effects}

A potentially important aspect of pressure ionization we have ignored is the onset of delocalization of the electronic wave functions at high density, leading to possible electronic "conduction bands" in the neutral mixture, similar to band narrowing in solids. Given the lack of exact calculations of such electron delocalization effect in a finite-temperature fluid, we have estimated the size of the effect in an ex post-facto calculation. We used zero-temperature band-gap calculations (Friedli and Ashcroft 1977). The fraction of electrons thermally excited into the conduction band varies from less than $10 \%$ at $8000 \mathrm{~K}$ to $2 \%$ at the critical point. Consequently we find the effect on the PPT to be small. Moreover, as discussed previously, the $\mathrm{H}_{2}$ molecule is likely to undergo substantial rotation at pressures characteristic of the PPT, which probably widens the band gap. 
Table 13.2. Comparison between the present model (lower row) and calculations based on the Density Functional formalism (DFT) of Perrot and Dharma-wardana (upper row) for hydrogen at high temperature and density. For each temperature-density point, we give the effective charge, the pressure and the entropy. The effective charge $Z^{*}$ denotes the average degree of ionization. (units are in CGS)

\begin{tabular}{lllll}
\hline \hline $\log T$ & $\log \rho$ & $Z^{*}$ & $\log P$ & $\log S$ \\
\hline 5.161 & -1.269 & 0.777 & 12.045 & 9.209 \\
& & 0.712 & 12.033 & 9.213 \\
& -0.4755 & 1.000 & 12.837 & 9.125 \\
& & 0.695 & 12.811 & 9.128 \\
& 0.4275 & 1.000 & 13.85 & 9.012 \\
& 1.330 & 0.883 & 13.826 & 9.009 \\
& & 1.000 & 15.182 & 8.899 \\
5.462 & -1.3455 & 1.000 & 15.169 & 8.879 \\
& & 0.923 & 12.313 & 9.264 \\
& -0.4755 & 0.812 & 12.296 & 9.263 \\
& & 1.000 & 13.169 & 9.178 \\
& 0.4275 & 0.781 & 13.154 & 9.178 \\
& & 1.000 & 14.104 & 9.071 \\
& 1.331 & 0.904 & 14.088 & 9.067 \\
& & 1.000 & 15.273 & 8.957 \\
6.06 & -1.375 & 1.000 & 15.263 & 8.943 \\
& & 0.992 & 12.908 & 9.344 \\
& & 0.938 & 12.893 & 9.340 \\
& -0.4755 & 1.000 & 13.802 & 9.269 \\
& & 0.910 & 13.787 & 9.266 \\
& 0.4275 & 1.000 & 14.702 & 9.179 \\
& \multirow{2}{*}{1.331} & 0.960 & 14.693 & 9.176 \\
& & 1.000 & 15.652 & 9.075 \\
& & 1.000 & 15.643 & 9.066 \\
\hline
\end{tabular}

\subsection{Other models}

Marley and Hubbard (1988) also computed a forced transition between a neutral and a fully ionized model. Compared to other efforts, their two models are closest to our own in level of accuracy and detail. Their transition line lies slightly above the one obtained in our calculations for the forced transition (see Fig.4). The difference arises from the cruder treatment of the internal levels of the molecules in Marley and Hubbard's calculations.

Ebeling and Richert published two calculations for the PPT. Their two models differ in detail but are very similar in spirit and give essentially the 
same critical point, at $T_{c}=16500 \mathrm{~K}, P_{c}=0.228 \mathrm{Mbar}$ and $\rho_{c}=0.13 \mathrm{gcm}^{-3}$. In the first model (Ebeling and Richert 1985a), atoms and molecules interact through hard-sphere potentials with fixed diameters, plus a Van-der-Waals correction. Molecules are approximated as two atoms in the hard-sphere free energy. Internal states are not included in the treatment and there is no coupling between charged and neutral particles. Their approximate coexistence curve crosses the experimental double-shock Hugoniot curve for deuterium, where no evidence is found for a PPT. This rules out the model, based on too crude approximations.

In a second paper (Ebeling and Richert 1985b), molecules are excluded from the model. Bound states are introduced in the form of the PlanckLarkin partition function. This has been demonstrated to be incompatible with a chemical picture (Däppen et al. 1987). They find a second critical point surprisingly close to their previous dtermination. Again this is incompatible with existing shock-wave experiments at high-temperature which must be reproduced by any model aimed at describing the EOS of dense fluid hydrogen. In particular, the critical pressure is lower than predicted by the model (9). This fact stems from the excessively repulsive hard-sphere potentials between neutral particles. These potentials are completely inapropriate in the density range where pressure ionization occurs.

\subsection{Effect of the PPT on the structure and the evolution of low-mass stars and giant planets}

The PPT predicted by our model occurs at densities and temperatures characteristic of giant planets and low-mass brown dwarfs. New interior models of Jupiter and Saturn including the PPT have been computed recently (Chabrier et al. 1992). Interior models assuming homogeneous $\mathrm{H} / \mathrm{He}$ envelopes (i.e. no PPT) could not satisfy the observational constraints, adding titillating support for the presence of a PPT between a molecular $\mathrm{H}_{2} / \mathrm{He}$ envelope and a metallic $\mathrm{H}^{+} / \mathrm{He}^{++}$envelope. The phase transition modifies the thermal structure of the planets, leading to a hotter internal adiabat than the one obtained with no PPT, because of the positive entropy jump at the PPT (see Table I).

The effect of the PPT on the evolution of Jupiter, Saturn and low-mass brown dwarfs has also been examined in detail (Saumon et al. 1992). For fully convective, adiabatic objects, the evolutionary timescale is given by :

$$
L d t=L_{s} d t-\int_{0}^{M}(T \delta S) d m
$$


for a time interval $d t . L$ is the luminosity of the planet, $L_{s}=4 \pi R^{2} \sigma T_{s}^{2}$ is the heating caused by solar radiation, and $S$ is the entropy per gram, including (or not) the entropy discontinuity at the PPT. The phase transition was found to alter the present age of Jupiter and Saturn by a few percent. The cooling of brown dwarfs is most strongly affected at the time when the interior adiabat crosses the critical point.

\subsection{Conclusion}

This review has focussed on an EOS of dense hydrogen suitable for astrophysical purposes, in particular for the interior of dense objects like giant planets and low-mass stars. Extension to the case of helium is under progress (Aparicio and Chabrier 1994). The proposed EOS is based on realistic interparticle potentials and a self-consistent treatment of the internal levels. The model reproduces experimental Hugoniots up to $P \approx 1 \mathrm{Mbar}$. In the domain of partial ionization, a complete model of the interacting fourcomponent $\left(\mathrm{H}_{2}, \mathrm{H}, \mathrm{H}^{+}, \mathrm{e}^{-}\right)$fluid is generated. The present model, based on the so-called chemical picture, represents the best compromise between the rigorous treatment of the $\mathrm{N}$-body problem, as in the physical picture, and the practical application. Preliminary comparisons between the two formalisms in the high-temperature limit of partially ionized hydrogen shows excellent agreement on the equation of state and the entropy, the two most important thermodynamic quantities used in stellar evolution. The model predicts a first-order phase transition between a mainly molecular phase and a partially ionized plasma. This model free energy leads to interior models for Jupiter and Saturn in better agreement with the observations.

\section{References}

Aparicio J.M. and Chabrier G., to appear in Phys. Rev. E; see also these proceedings.

Ashcroft N.W. and Stroud D., Solid State Phys., 33, Academic, New-York (1978)

Chabrier G., J. de Physique France 51, 1607, (1990)

Chabrier G., Saumon D., Hubbard W.B., and Lunine L.I., Ap. J. 391, 817, (1992)

Däppen W., Anderson L. and Mihalas D., Ap. J., 319, 195, (1987)

DeWitt H.E., J. Math. Phys. 9, 1216, (1962)

Ebeling W. and Richert W., Phys. Lett. A, 108, 80, (1985a)

Ebeling W. and Richert W., Phys. Status Solidi, 128, 467, (1985b)

Friedli C. and Ashcroft N.W., Phys. Rev. A, 16, 662, (1977)

Goldstein R.E. and Ashcroft N.W., Phys. Rev. Lett., 55, 2164, (1985)

Grabowski B, Madej J. and Halenka J, Ap.J., 313, 750, (1987)

Gründke E.W. and Henderson D., Mol. Phys. 24 (2), 269, (1972)

Hashimoto S. and Yamaguchi N., Phys. Lett. A, 95, 299, (1983) 
Huber K.P. and Herzberg G., Molecular Spectra and Molecular Structures, (Van Nostrand, Princeton), (1979)

Hemley R.J. and Mao H.K., Phys. Rev. Lett., 61, 857, (1988)

Hemley R.J., Mao H.K. and Shu J.F., Phys. Rev. Lett, 65, 2670, (1990)

Hemley R.J., Mao H.K., Finger L.W., Jephcoat A.P., Hazen R.M. and Zha C.S., Phys. Rev. B, 42, 6458, (1990)

Hümmer D.G. and Mihalas D., Ap. J. 331, 794, (1988)

Ichimaru S., Iyetomi H. and Tanaka S., Phys. Rep., 149, (1987)

Kang H.S, Lee C.S., Ree T. and F. Ree J. Chem. Phys. 82 (1), 414, (1985)

Kollos W. and Wolniewicz, J. Chem. Phys., 43, 2429, (1965)

Kraeft W.D., Kremp D., Ebelimg W. and Röpke G., Quantum Statistics of Charged Particle Systems, Plenum (1986)

Mansoori G.A., Carnahan N.F., Starling K.E. and Leland T.W. J. Chem. Phys. 54 (4), 1523, (1971)

Marley M.S. and Hubbard W.B., Icarus, 73, 53, (1988)

Nellis W.J., Holmes N.C., Mitchell A.C., Trainor R.J., Governo G.K., Ross M. and Young D.A., Phys. Rev. Letters 53 (13), 1248, (1984)

Porter R.N. and Karplus M., J. Chem. Phys., 40, 1105, (1964)

Redmer R., Röpke G and Zimmermann R., J. Phys. B, 20, 4069, (1987)

Ross M, Ree F.H. and Young D.A., J. Chem. Phys., 79, 1487, (1983)

Saumon D. and Chabrier G., Phys. Rev. A 44, 5122, (1991)

Saumon D. and Chabrier G., Phys. Rev. A 46, 2084, (1992)

Saumon D., Chabrier G. and Weis J.J., J. Chem. Phys., 90, 7395, (1989)

Saumon D., Hubbard W.B., Chabrier G. and Van Horn H.M., Ap. J. 391, 827, (1992)

Stevenson D.J. and Salpeter E.E., Ap. J. Suppl., bf 95, 221, (1977)

Utsumi K. and Ichimaru S., Phys. Rev. A, 26, 603, (1982)

Weeks J.D., Chandler D. and Andersen H.C., J. Chem. Phys. 54, 5237, (1971)

Weise W.L., Kelleher D.E. and Paquette D.R., Phys. Rev. A, 6, 1132, (1972)

Wigner E. and Huntington H.B., J. Chem. Phys., 3, 764, (1935) 